\title{
Analysis of surveillance position error for airfield detection
}

\author{
SungKrwan Ku \\ Department of Aviation Industrial and System Engineering, Hanseo University, Taean, Republic of Korea \\ Hojong Baik \\ School of Air Transport, Transportation and Logistics, \\ Korea Aerospace University, Goyang, Republic of Korea, and \\ Taehyoung Kim \\ Ramsey County Public Works, Arden Hills, Minnesota, USA
}

\begin{abstract}
Purpose - The surveillance equipment is one of the most important parts for current air traffic control systems. It provides aircraft position and other relevant information including flight parameters. However, the existing surveillance equipment has certain position errors between true and detected positions. Operators must understand and account for the characteristics on magnitude and frequency of the position errors in the surveillance systems because these errors can influence the safety of aircraft operation. This study aims to develop the simulation model for analysis of these surveillance position errors to improve the safety of aircrafts in airports.

Design/methodology/approach - This study investigates the characterization of the position errors observed in airport surface detection equipment of an airport ground surveillance system and proposes a practical method to numerically reproduce the characteristics of the errors.

Findings - The proposed approach represents position errors more accurately than an alternative simple approach. This study also discusses the application of the computational results in a microscopic simulation modeling environment.

Practical implications - The surveillance error is analyzed from the radar trajectory data, and a random generator is configured to implement these data. These data are used in the air transportation simulation through an application programing interface, which can be applied to the aircraft trajectory data in the simulation. Subsequently, additionally built environment data are used in the actual simulation to obtain the results from the simulation engine.

Originality/value - The presented surveillance error analysis and simulation with its implementation plan are expected to be useful for air transportation safety simulations.
\end{abstract}

Keywords Simulation, Air traffic control system, Air transportation, Airport surface detection equipment, Surveillance position error

Paper type Research paper

\section{Introduction}

Current air traffic control (ATC) systems heavily rely on surveillance equipment that continuously provides the positions and other relevant information of an aircraft and its flight parameters. For example, the advanced surface management guidance and control system (A-SMGCS) controls aircraft movements on the airfield by using the position information from the airport surface detection equipment (ASDE), a ground surveillance system (Campbell et al., 2014; European Organization for Civil Aviation Equipment, 2015). However, the existing surveillance equipment is not perfect. Certain position errors exist, that is, the difference between the true and detected positions. The magnitude and frequency of the position errors are the critical factors determining the performance and reliability of any ATC system. Thus, operators must understand and account for the

The current issue and full text archive of this journal is available on Emerald Insight at: www.emeraldinsight.com/1748-8842.htm

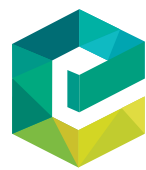

Aircraft Engineering and Aerospace Technology 90/6 (2018) 962-966

Emerald Publishing Limited [ISSN 1748-8842] [DOI 10.1108/AEAT-09-2017-0207] characteristics of the position errors in the surveillance systems when assessing the effectiveness and safety of an existing or new ATC system, particularly, when the assessment includes numerical simulations that portray each flight individually.

Analysts can reproduce real-world conditions and assess the effect of proposed concepts or changes to existing systems at low cost and, more importantly, with zero risk using surveillance simulation models. Therefore, microscopic simulation is widely used as an analytical tool for air transportation studies, such as new concept design, impact

\footnotetext{
(C) SungKwan $\mathrm{Ku}$, Hojong Baik and Taehyoung Kim. Published by Emerald Publishing Limited. This article is published under the Creative Commons Attribution (CC BY 4.0) licence. Anyone may reproduce, distribute, translate and create derivative works of this article (for both commercial \& non-commercial purposes), subject to full attribution to the original publication and authors. The full terms of this licence may be seen at http://creativecommons.org/licences/by/4.0/legalcode
}

This research was supported by a grant (18CTAP-C129733-02) from Technology Advancement Research Program (TARP) funded by Ministry of Land, Infrastructure and Transport of Korean government.

Received 21 September 2017

Revised 26 December 2017

4 January 2018

Accepted 20 January 2018 
analysis, and safety analysis. However, the level of reality in the simulation varies with the adopted model and the accuracy of the input data. The range of the input items showing random characteristics varies depending on their similarity with the actual situation, which influences the simulation reliability. Therefore, the generation and analysis of suitable input data are crucial for ensuring the high reliability of experimental results.

This study investigates the characterization of the position errors observed in ASDE and analyzes the surveillance data characteristics among the input data required for air transportation simulation. This study also discusses the results computed from the adjusted simulation model and suggests methods to enhance the simulation accuracy.

\section{Air transportation simulation and surveillance error for safety research}

\section{Overview of air transportation simulation and surveillance position error}

Air transportation simulation is the process of reproducing and calculating the movement of aircraft in airspace and airports. Factors that can be measured during an actual aircraft operation, such as travel time, delay time, and fuel consumption, are generally the analysis targets. Aircraft transportation routes, including air routes, airport locations and airfield locations, are reproduced. General air transportation simulations use a modeled pop-up time (i.e. schedule time) and aircraft performance to perform quick calculations on a computer. The physical forms of the airspace and the airport, as well as the stochastic characteristics of flight trajectories that occur under conditions of uncertainty, such as aircraft performance, atmosphere and temperature, are required for a realistic and accurate air transportation simulation.

A radar installed near the airport is generally used to monitor the position and the movement of aircraft in the airspace and airports. The primary radars can monitor aircraft by analyzing the time required for radio waves transmitted from a rotating radar antenna to return after being reflected by an aircraft. Aircraft surveillance can only be performed in intervals of the order of a few seconds because of the operational limitations of current radars. The detected positions may occasionally appear to be scattered even if the target aircraft is stationary because of the variation in the relative positions of the reflected waves. The magnitude of these errors varies depending on whether the ground or the airspace is being monitored and increases with the increase in the distance between the aircraft and the radar. The automatic dependent surveillance-broadcast (ADS-B) technology is considered the next-generation of air surveillance technology because it enables an aircraft to transmit its position information to nearby aircraft or control towers (ICAO, 2004 and $\mathrm{Ku}$ and Baik, 2015). The position information measured by the global navigation satellite system (GNSS), such as global positioning system data, is mainly used to realize this function. The GNSS provides relatively accurate position information compared with radar technology. The precision can be further enhanced using additional correction measures. However, the ADS-B technology is completely dependent on the aircraft for the position information, and ATC facilities cannot directly manage the stage of position information generation, which results in an inconsistent reliability of the obtained information (Schuster and Ochieng, 2011; Ju et al., 2014).

\section{Air transportation simulation and surveillance for safety analysis}

A high level of safety management is required in the aviation industry because even a single accident has a great impact. Therefore, the measurement of aviation safety, including planning, operation, and post-evaluation, is an important concern in the field of air transportation. Many studies are currently investigating the use of simulation as a substitute for actual operation experiments in safety assessment, which necessitate a substantial amount of time and resources.

Appropriate surveillance functions are essential for achieving a safe and efficient aircraft operation in airspace and airports. The US Federal Aviation Administration, which developed ASDE-X, is researching on improving the surveillance accuracy to ensure a safe and efficient airport operation (Sandberg, 2012). The A-SMGCS, which is an integrated support system for safe and efficient airport management, has been used in Europe, and surveillance functions with high precision are planned under this system. However, actual operational data is required to verify the effectiveness of such systems. Simulation is an effective alternative that can overcome this drawback. Scholars at MIT have identified the following steps and conditions for realizing a fast air transportation simulation. Data on the surveillance error, which varies with the airport and equipment, are included as a condition to verify the safety level (Campbell et al., 2014).

\section{Surveillance trajectory analysis}

Airfield surveillance trajectory data

ASDE is a type of radar that provides target information to the air traffic controller by detecting aircraft and vehicle movements in the airfield. ASDE has high precision, but its detection range is smaller than that of the radar that monitors the airspace around the airport. The surveillance target accuracy of ASDE varies with various factors, such as the distance between the aircraft and antenna, area where the radio wave is reflected off the aircraft, and weather conditions. Therefore, position errors can occur in ASDE even when the target object is completely stationary, such as a stationary aircraft waiting to enter a runway. These errors can cause a corresponding error in runway holding positions (i.e. the minimum separation between aircraft approaching a runway). Consequently, a violation alarm may be triggered even when the aircraft is safe and maintaining the regulated separation or an actual violation may not be registered by the system.

In this study, the ASDE trajectories of a stationary aircraft at the holding position of runway 07 at Jeju International Airport, which is an operating airport in South Korea (Figure 1), were analyzed to characterize the surveillance position errors. A total of 10,380 trajectories from 103 aircraft were analyzed.

\section{Trajectory analysis}

The lateral distribution of the aircraft position was recorded as the left and right separation distance of the trajectories based 
Figure 1 Jeju airport runway 07 holding position and stop trajectory analysis area

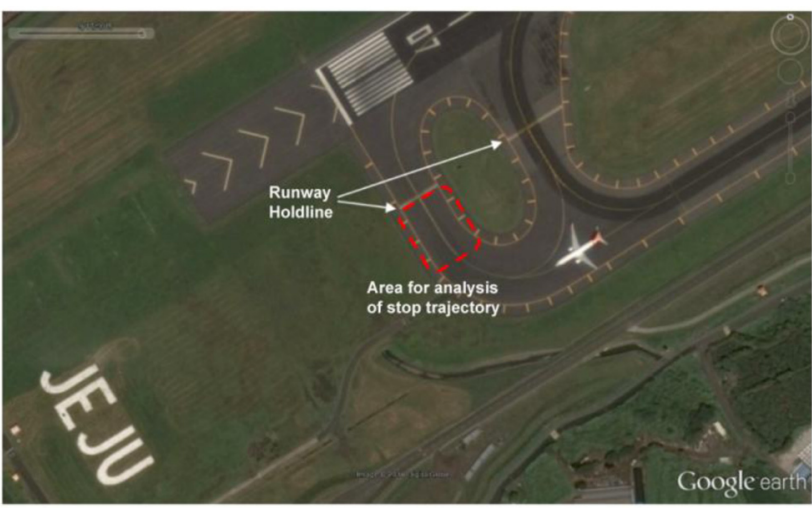

on the taxiway centerline. The trajectories located on the left were set as negative numbers $(-)$, while those on the right were set as positive numbers $(+)$. The longitudinal distribution was recorded as the trajectory position based on the runway holding position, with negative numbers (-) corresponding to the trajectories that did not pass through the runway holding position and positive numbers $(+)$ corresponding to those that did. It is possible to make the lateral and longitudinal errors when pilots do not follow exactly the taxiway centerline or the holding position. But, surveillance errors already include these errors.

The trajectory lateral mean value was $-12.18 \mathrm{~m}$; the standard deviation was $2.9 \mathrm{~m}$; and the maximum and minimum values were $7.16 \mathrm{~m}$ and $-21.81 \mathrm{~m}$, respectively. The aircraft trajectory position was biased to the left of the taxiway centerline. The longitudinal mean value was $-38.15 \mathrm{~m}$; the standard deviation was $10.13 \mathrm{~m}$; and the maximum and minimum values were $11.84 \mathrm{~m}$ and $-67.79 \mathrm{~m}$, respectively. Most trajectory positions did not pass through the runway holding position. However, a few surveillance trajectories exceeded the runway holding position in the stop condition.

\section{Simulation and application of the surveillance position error}

\section{Airfield surveillance trajectory data simulation with position error}

The lateral and longitudinal surveillance errors must be simultaneously applied to simulate the surveillance error of the stationary aircraft. Two-dimensional (2D) position errors can be complexly applied using multivariate normal distribution. The drawback of this method, however, is the difficulty of implementing tail values, which are present in the actual data. Therefore, principal component analysis (PCA) and kernel density estimation were used to generate the tail values (Figure 2). First, the observation data was subject to PCA, following which the angle of the principal component axis was measured. Second, the 2D $x$-axis and $y$-axis data were generated through kernel density estimation, following the data that rotated as much as the measured PCA angle. Finally, the lateral and longitudinal $2 \mathrm{D}$ position data were re-rotated to the original angle. MATLAB was used for the simulation and the data analysis.
Figure 2 Data simulation through PCA

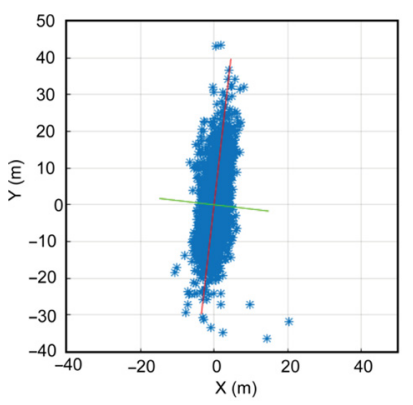

(a)

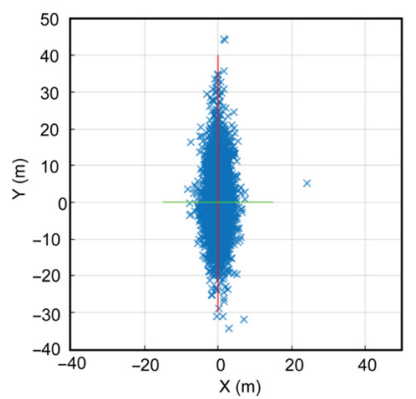

(c)

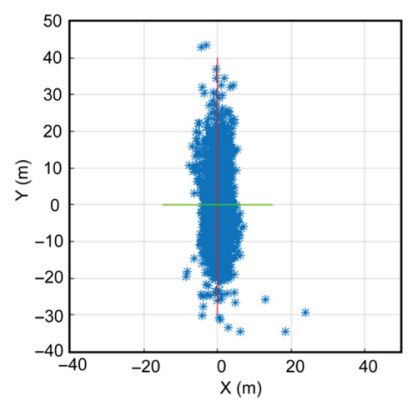

(b)

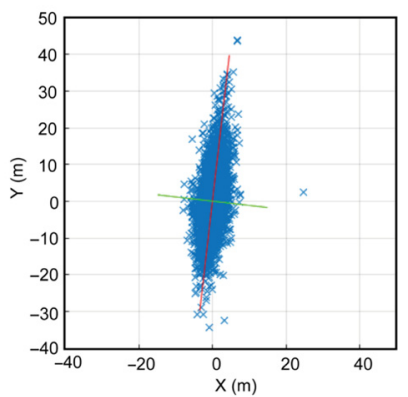

(d)
Notes: (a) Observation data and PCA; (b) rotate the observation data; (c) simulated data of kernel density estimation; (d) rotate the simulation data

Figure 3 presents the ASDE-observed data (left), the simulated position data that only follows multivariate normal distribution (center) and the simulated position data subjected to complexly applied PCA and kernel density estimation (right). The ASDEobserved data are the actual observation value, including the surveillance position error, of each stopped aircraft. A simulation that followed multivariate normal distribution based on this data and a simulation that complexly followed the normal distribution and kernel density estimation were performed.

Compared with the simulation performed using multivariate normal distribution, the ASDE observation values generated in the simulation data that complexly used the PCA and kernel density estimation were closer in form to the observed data. The simulation obtained from multivariate normal distribution with PCA and kernel density estimation was more similar to that obtained from the ASDE surveillance data than that obtained from multivariate normal distribution.

\section{Goodness of fit test for the airport surface detection equipment-observed and simulated data}

The chi-square goodness-of-fit test was conducted to verify whether the data generated by the simulation could properly implement the small tail value characteristics observed in the actual data. The absolute distance from the center point $(0.0)$ of each data location was set as the main measurement variable. In all, $5 \mathrm{~m}$ of distance was set for each rank, and a tail-part distance of $24.1 \mathrm{~m}$ or longer was set as one rank to deduct the minimum value of the nominal scale variable for the chi-square 
Figure 3 Position data with the ASDE-observed and simulated errors
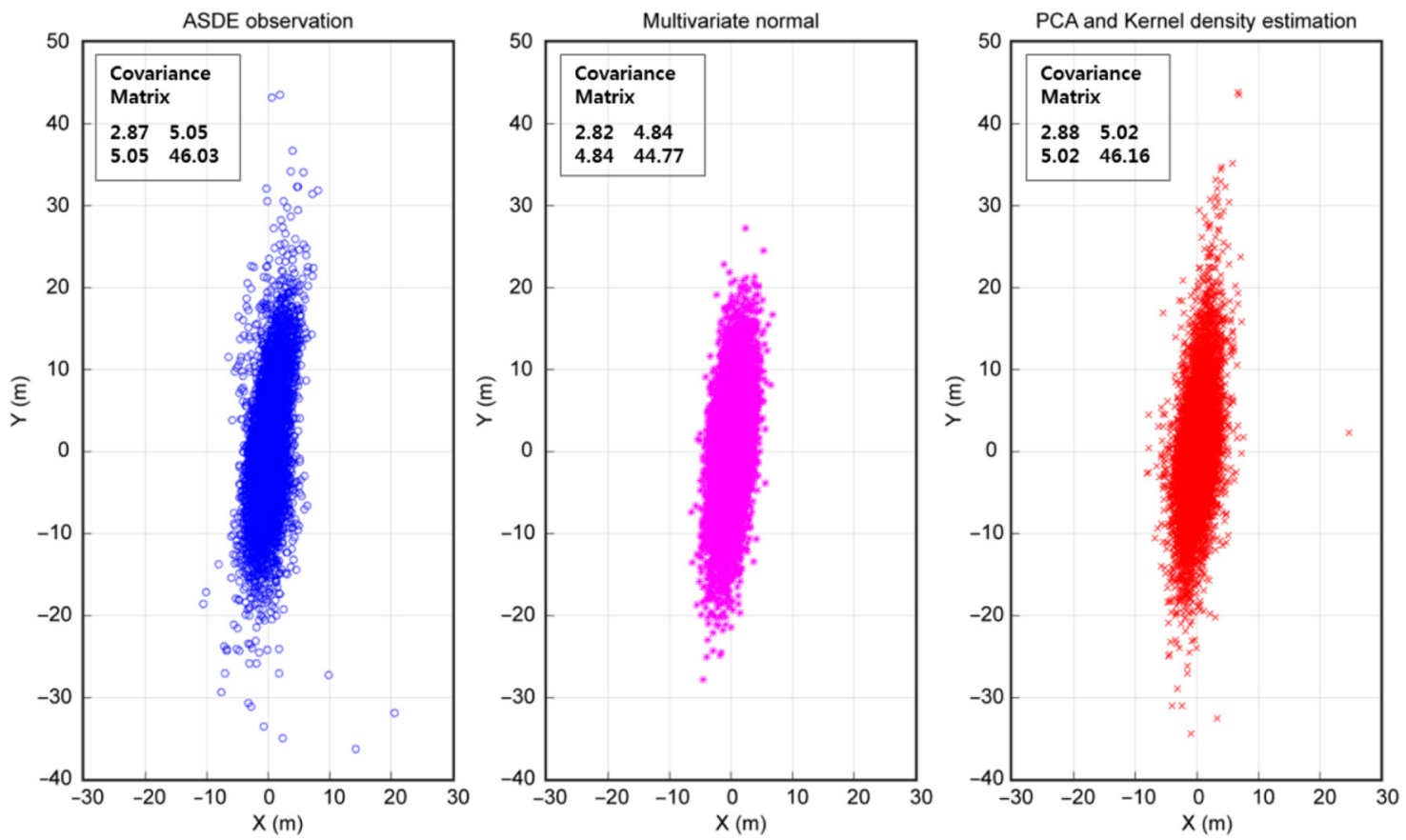

Table I Expected and simulated frequency and result of the chi-square test for goodness of fit

\begin{tabular}{lccr}
\hline Distance $(\mathrm{m})$ & $\begin{array}{c}\text { Observation } \\
\text { data }\end{array}$ & $\begin{array}{c}\text { Multivariate } \\
\text { normal } \\
\text { distribution }\end{array}$ & $\begin{array}{r}\text { PCA and } \\
\text { kernel } \\
\text { estimation }\end{array}$ \\
\hline 0 to $<5$ & 6117 & 5,328 & 6,011 \\
5 to $<10$ & 2795 & 3,521 & 2,891 \\
10 to $<15$ & 1063 & 1,232 & 1,067 \\
15 to $<20$ & 296 & 263 & 298 \\
20 to $<24.1$ & 56 & 31 & 61 \\
24.1 or longer & 53 & 5 & 52 \\
Total & 10,380 & 10,380 & 10,380 \\
Content & & Chi-square & $H_{0}$ result \\
ASDE observation versus & & & \\
simulation using multivariate & 774.82 & $\mathrm{X}$ \\
normal distribution & & 5.52 & 0 \\
ASDE observation versus & & & \\
PCA and kernel density estimation & &
\end{tabular}

test to be 5 or higher (Table I). The mean of 30 iterations of the simulated data was rounded to the nearest tenth.

Table I shows the chi-square test results for the ASDEobserved and simulated data. Here, the null hypothesis is as follows:

$H_{0}$. Simulation data follow the observed data.

The alternative hypothesis is as follows:

$H_{1}$. Simulation data do not follow the observed data.

The chi-square value of the simulation data obtained considering multivariate normal distribution was 774.82 , which was significant $(P=0.05$ and $\mathrm{Df}=5)$. Therefore, this simulation data cannot be used because $H_{0}$ was rejected. In contrast, the chi-square value of the simulation data through kernel density estimation and PCA was 5.52, which was non-significant $(P=0.05$ and $\mathrm{Df}=5)$. Thus, $H_{O}$ holds, indicating the appropriate implementation of the ASDE observation values with the simulation data.

Figure 4 Architecture of the air transportation simulation with the surveillance position error

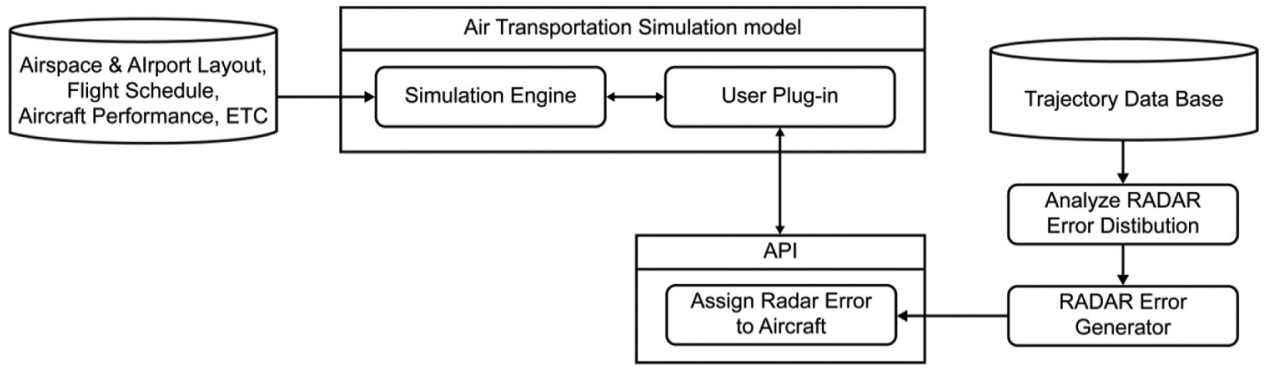




\section{Suggested applications}

Figure 4 shows the architecture of the air transportation simulation, where the surveillance position error is applied. The surveillance error was analyzed from the radar trajectory data, and a random generator was configured to implement these data. These data were used in the air transportation simulation through an application programing interface, which can be applied to the aircraft trajectory data in the simulation. Subsequently, additionally built environment data were used in the actual simulation to obtain the results from the simulation engine. Our study also can be used for the safety computation simulation to supplement the shortcomings of risk analysis that needs a long time using statistically historical data of operational results.

\section{Conclusion}

Surveillance data have position errors between the true and detected positions although current ATC systems depend on aircraft surveillance data. This study investigates the characterization of the position errors observed in ASDE of an airport ground surveillance system and proposes a practical method to numerically reproduce the characteristics of the errors in a microscopic simulation modeling environment. The simulation results of proposed approach represent position errors more accurately than an alternative simple multivariate approach. The chi-square test shows that our simulation data for surveillance position error are statistically significant. The architecture of air transportation simulation is also suggested to represent the surveillance position error for operation of airports. The presented surveillance error analysis and simulation with its implementation plan are expected to be useful for air transportation safety simulations.

\section{Further work}

This study considers the surveillance error analysis for ranges in runway holding position, and its application using an integrated simulation model will be investigated in the future study. The calibration results of surveillance position error would be different for other scenarios because these results can be determined by various parameters such as the location of radar, distance of aircraft from radar, size of aircraft, direction of aircraft and so on. The following study will consider more various parameters under other areas of the airport and other airports.

\section{References}

Campbell, S.D., Londner, E., Keefe, J. and Edwards, C. (2014), "Airport surveillance requirements validation using monte carlo simulation", Digital CommunicationsEnhanced Surveillance of Aircraft and Vehicles, Tyrrhenian International Workshop, pp. 29-34.

European Organization for Civil Aviation Equipment (2015), "Minimum aviation system performance specification for advanced surface movement guidance and control systems (A-SMGCS) level 1 and 2", available at: www.icao.int/ Meetings/anconf12/Document\%20Archive/9830_cons_en[1]. pdf (accessed 1 September 2017).

International Civil Aviation Organization (2004), "Advanced surface movement guidance and control systems (A-SMGCS) manual", available at: http://standards.globalspec.com/std/ 9935945/eurocae-ed-87 (accessed 1 September 2017).

Ju, Y.H., Ku, S.K. and Hong, K.Y. (2014), "A study for avoidance alarm algorithm with ADS-B message", fournal of Advanced Navigation Technology, Vol. 19 No. 5, pp. 379-388.

$\mathrm{Ku}, \mathrm{S} . \mathrm{K}$. and Baik, H.J. (2015), "Analysis of ADS-B ground trajectory data using non-aviation approval public data", fournal of the Korean Society for Aviation and Aeronautics, Vol. 23 No. 4, pp. 6-11.

Sandberg, M. (2012), "Applications of ASDE-X data to the analysis of airport surface operations", available at: https:// dspace.mit.edu/handle/1721.1/74469\#files-area (accessed 1 September 2017).

Schuster, W. and Ochieng, W. (2011), “Airport surface movementcritical analysis of navigation system performance requirements", foumal of Navigation, Vol. 64 No. 2, pp. 281-294.

\section{Corresponding author}

Taehyoung Kim can be contacted at: thkim@vt.edu

For instructions on how to order reprints of this article, please visit our website:

www.emeraldgrouppublishing.com/licensing/reprints.htm

Or contact us for further details: permissions@emeraldinsight.com 